\title{
Hallazgos de tomografía computarizada en coenurosis cerebral crónica con hidrocéfalo secundario en una oveja joven
}

\author{
Computed tomographic findings in chronic cerebral coenurosis associated with secondary \\ hydrocephalus in a young ewe \\ M Gómez ${ }^{\mathrm{a}}$, N Tadich $^{\mathrm{b}}$, M Mieres ${ }^{\mathrm{b}}$, H Bustamante ${ }^{\mathrm{b}}$, J Galecio ${ }^{\mathrm{b}}$, M Hervéc \\ anstituto de Anatomía Veterinaria, Facultad de Ciencias Veterinarias, Universidad Austral de Chile, Valdivia, Chile. \\ bInstituto de Ciencias Clínicas Veterinarias, Facultad de Ciencias Veterinarias, Universidad Austral de Chile, Valdivia, Chile. \\ 'Instituto de Ciencia Animal y Tecnología de Carnes, Facultad de Ciencias Veterinarias, Universidad Austral de Chile, Valdivia, Chile.
}

\section{SUMMARY}

The purpose of this study was to describe the computed tomographic (CT) features of a 14 month old ewe showing clinical signs of cerebral coenurosis. The CT analysis of the head was made using a fourth generation CT scanner. On transverse CT images the cyst was seen as an hipodense, ring enhanced mass located in the cerebral hemispheres and ventricular system. Ventriculomegaly and atrophy of the cortical tissue was also observed indicating the presence of secondary hydrocephalus. The Coenurus was surgically removed by skull trepanation through the frontal bone and after a few days the animal recovered its healthy status. CT proved to be useful in demonstrating the anatomic location of the cyst and was helpful in surgical planning.

Palabras clave: coenurosis, tomografía computarizada, ovinos.

Key words: coenurosis, computed tomography, sheep.

\section{INTRODUCCION}

La coenurosis cerebral en ovinos es una neuroparasitosis producida por el estado larval del metacestodo Taenia multiceps al invadir el tejido cerebral o la médula espinal (Soulsby 1982, Martin y Aitken 2000). La infestación intestinal de los ovinos ocurre al ingerir pasturas o agua que contengan heces de perro contaminadas con el metacestodo (Soulsby 1982). La migración de la(s) larva(s) oncosfera hacia el Sistema Nervioso Central ocurre por vía hematógena y el desarrollo del quiste hasta producir signología clínica puede demorar de 2 a 8 meses (Martin y Aitken 2000). Esta enfermedad también ha sido descrita, aunque con menor frecuencia, en caprinos, bovinos, camélidos, gacelas, búfalo, equinos, gamuzas, conejos, gatos, perros, monos y seres humanos (Brewer 1983, Smith 1988, Yoshino y Momotani 1988, Fraser 1991, Lavin y col 1995, Ing y col 1998). Normalmente un completo examen neurológico es suficiente para un diagnóstico de coenurosis cerebral ovina crónica; sin embargo, la signología clínica puede confundirse con otras alteraciones neurológicas (Oliver 1987, Mayhew 1989, Achenef y col 2000, Komnenou y col 2000). Los signos, aunque variables, incluyen trastornos

Aceptado: 17.10.2006.

* Casilla 567, Valdivia, Chile; marcelogomez@uach.cl conductuales, depresión, convulsiones, aislamiento del rebaño, pérdida del apetito, paresis o parálisis uni o bilateral, inclinación de la cabeza, déficit posturales, ataxia, torneo, dolor a la palpación craneana, tremores musculares, hiperexcitabilidad, alteraciones visuales, alteraciones vestibulares y/o cerebelares, decúbito y muerte (Dyson y Linklatter 1979, Skerritt y Stallbaumer 1984). Técnicas como la radiografía simple (Tirgari y col 1987), ultrasonografía de cráneo (Doherty y col 1989º y más recientemente la tomografía computarizada (TC) (Gonzalo-Orden y col 1999) y la resonancia magnética (RM) (Gonzalo-Orden y col 2000) de la región cefálica han sido utilizadas en el diagnóstico, no obstante los reportes de estos últimos métodos son escasos. La TC de cráneo ha sido utilizada también en humanos y felinos con coenurosis cerebral (Smith y col 1988). La TC y la RM permiten obtener la localización exacta y determinar el tamaño y número de quistes en el encéfalo. Esto adquiere especial importancia en el planeamiento quirúrgico para la remoción del quiste parasítico. El presente trabajo reporta una descripción tomográfica de una coenurosis cerebral ovina que adicionalmente presentó hidrocéfalo no comunicante.

\section{MATERIAL Y METODOS}

Una hembra ovina, de 14 meses de edad, cruza Austral-Romney, de $35 \mathrm{~kg}$ de peso fue ingresada al Hospital 
Veterinario de la Universidad Austral de Chile en noviembre del 2005 por sintomatología de temblores, marcha en círculo, incoordinación y aparente ceguera. Al examen neurológico el animal evidenció ataxia, hemiparesis izquierda, ausencia del reflejo de amenaza en el lado izquierdo e inclinación de la cabeza hacia el lado derecho, torneo hacia el lado derecho y visión disminuida del ojo derecho. Adicionalmente se observó reblandecimiento óseo del hueso frontal izquierdo a nivel del botón córneo. Neuroanatómicamente la lesión se localizó a nivel de prosencéfalo con compromiso principal a nivel del hemisferio cerebral derecho. Diagnósticos diferenciales incluyeron coenurosis cerebral, listeriosis, hidrocefalia secundaria y absceso cerebral. Como exámenes complementarios se realizaron ultrasonografía y TC de cráneo.

La ultrasonografía a nivel de huesos frontales evidenció una zona anecoica a nivel izquierdo recubierta por una banda hiperecoica con características de cápsula. Ventral a la zona anecoica se observó tejido encefálico desplazado hacia el piso de la cavidad craneana. A nivel del lado derecho también se observó una zona anecoica correspondiente con densidad de líquido pero de menor tamaño. Ambas vistas ultrasonográficas sin embargo no permitieron diferenciar si se presentaba una sola cavidad quística o varias. Posteriormente, al examen ultrasonográfico, el animal fue examinado por TC.

Previamente se realizó un examen preanestésico al ovino, otorgándole una categoría ASA III. El animal fue premedicado con xilacina y atropina en dosis de $0,1 \mathrm{mg} / \mathrm{kg}$ y $0,02 \mathrm{mg} / \mathrm{kg}$, respectivamente, ambas administradas por vía intramuscular. Se introdujo una cánula en la vena cefálica izquierda y esta fue mantenida viable para administrar suero ringer lactato isotónico a una dosis de infusión de $10 \mathrm{ml} / \mathrm{kg} / \mathrm{h}$. Se indujo anestesia general mediante una combinación anestésica endovenosa, compuesta por ketamina en dosis de $6 \mathrm{mg} / \mathrm{kg}$ y diazepam en dosis de $0,5 \mathrm{mg} / \mathrm{kg}$. El animal fue mantenido en decúbito esternal, a fin de evitar aspiración por regurgitación. Una vez conseguida la inconciencia del animal fue intubado y mantenida la anestesia con isoflurano en oxígeno. Durante la TC el vaporizador se mantuvo al $2 \%$ y flujo de oxígeno a $2 \mathrm{~L} / \mathrm{min}$. Adicionalmente, durante el procedimiento se monitoreó con un oxímetro de pulso (Pulse Oximeter, Nonin Medical, INC., Model 8500AV, MN, USA.)

Las imágenes fueron obtenidas utilizando un escáner de cuarta generación (IQ/PQ 4.25 Diagnostics, Picker International, Ohio, USA). El examen tomográfico no contrastado fue realizado con el animal posicionado en decúbito esternal y con las extremidades torácicas extendidas cranealmente y las pélvicas extendidas caudalmente. La cabeza fue fijada al soporte metálico de la camilla del equipo mediante cinta adhesiva. Imágenes transversales fueron obtenidas a nivel de cráneo desde la lámina cribosa del etmoides hasta el foramen magno utilizando para ello una radiografía digital lateral inicial o topograma. La adquisición se realizó sin contraste y mediante cortes contiguos de $2 \mathrm{~mm}$ de grosor y $2 \mathrm{~mm}$ de intervalo. Se obtuvieron además reconstrucciones multiplanares, dorsales y sagitales. Las imágenes fueron presentadas utilizando una ventana para tejido blando (WW: 216 unidades Hounsfield; WL: 79 unidades Hounsfield) y otra para hueso (WW: 1575 unidades Hounsfield; WL: 753 unidades Hounsfield). Parámetros técnicos además incluyeron $130 \mathrm{Kv}$ y 85 mA.

\section{RESULTADOS Y DISCUSION}

El examen tomográfico evidenció en la fosa caudal una zona hipodensa esférica e irregular, de posición intraaxial, de paredes delgadas y nítidas, $2 \mathrm{~cm}$ de diámetro, no asociada a edema o efecto de masa y de contenido homogéneo e isodenso al líquido cefalorraquídeo (figura 1). La estructura quística además presentó un anillo hiperdenso incompleto (figura 1). A nivel de fosa media se encontró aumento de tamaño de los ventrículos laterales y tercer ventrículo y atrofia severa de hemisferios cerebrales observado tanto en las imágenes transversales (figura 2) como en las reconstrucciones longitudinales (figura 3). A este nivel fue difícil observar la delimitación entre la estructura quística y los ventrículos laterales debido a la igual densidad entre el quiste y el líquido cefalorraquídeo. Zonas hiperdensas asociadas a las áreas radiolúcidas fueron observadas y fueron interpretadas como calcificaciones y/o acúmulos de protoescolices asociados a la pared del quiste (Gonzalo-Orden y col 1999).

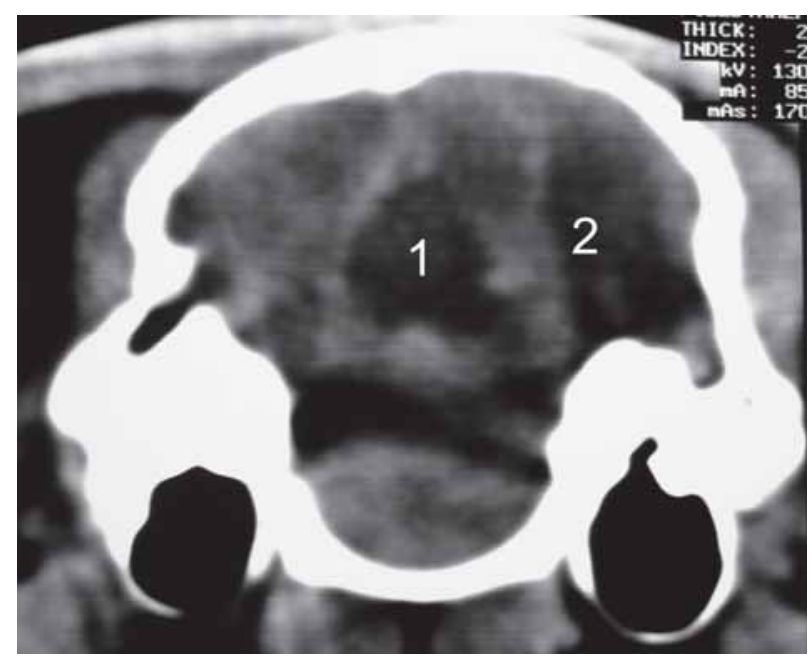

Figura 1. Imagen transversal de TC sin contraste de cráneo de oveja a nivel de fosa caudal. Observe la masa hipodensa circular con bordes hiperdensos irregulares indicando la presencia del quiste (1) y dilatación ventricular a nivel del cuerno temporal izquierdo (2).

Non-contrast transverse CT image from a sheep skull at the caudal fossa. Notice the hipodense circular mass with irregular hiperdense borders indicating the cyst (1) and a ventricular dilatation in the left temporal horn (2). 


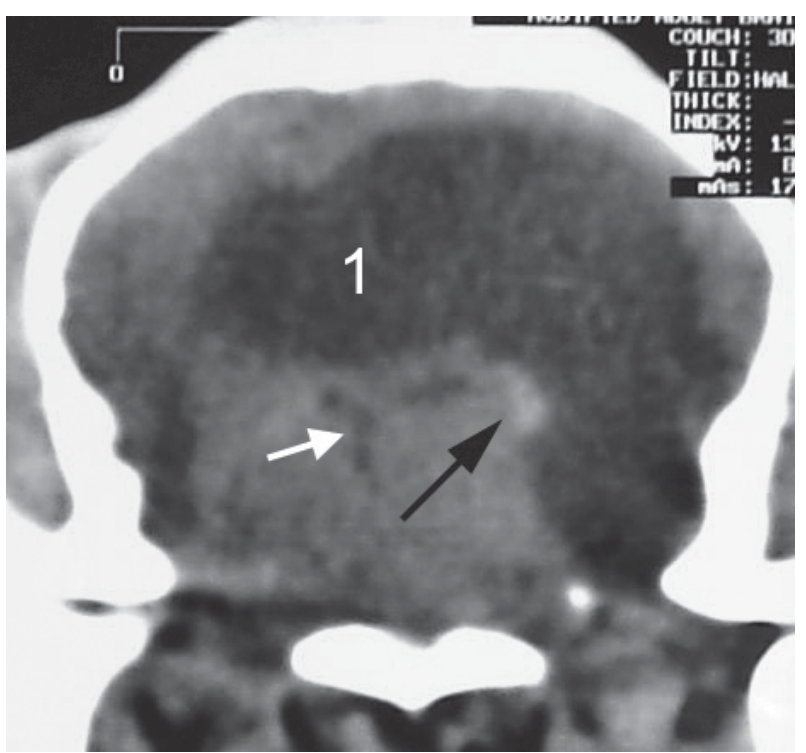

Figura 2. Imagen transversal de TC sin contraste de cráneo de oveja a nivel de fosa media. Ventriculomegalia a nivel de ventrículo lateral izquierdo (1) y dilatación de tercer ventrículo (flecha blanca). No es posible sin embargo distinguir los límites del quiste parasitario. Adicionalmente se observa una región hiperdensa sobre epitálamo izquierdo y dentro del quiste que correspondería a la presencia de los escólices del quiste (flecha negra).

Non-contrast transverse CT image from a sheep skull at the medium fossa. Ventriculomegaly in the left lateral ventricle (1) and dilation of the third ventricle (white arrow). However, it is not possible the limits of the parasitic cyst. Additionally an hyperdense region is observed over the left epithalamus and inside the cyst corresponding to cyst escolex (black arrow).

Posterior al examen de TC, el animal fue tratado quirúrgicamente para la remoción del quiste. La aproximación quirúrgica cerebral se realizó inicialmente en el hueso frontal izquierdo; sin embargo, no fue posible puncionar el quiste, por lo que se optó por una aproximación quirúrgica derecha mediante la técnica descrita (Skerrit y Stallbaumer 1984, 1986, Skerrit y Tadich 1986). Luego de la remoción quirúrgica completa del quiste, el animal presentó un aumento paulatino de la presión arterial directa por fuera de los rangos fisiológicos para la especie por aproximadamente 20 minutos. En el lapso de dos semanas posterior a la cirugía el animal recuperó paulatinamente sus funciones motoras mostrando finalmente una ataxia leve.

La coenurosis ovina constituye una patología de distribución geográfica mundial y de presentación endémica en ciertas regiones de Chile (Gutiérrez y col 1992). Si bien el diagnóstico clínico de coenurosis puede presentar algunas complicaciones, protocolos de examen neurológico diseñados específicamente para esta patología pueden ser suficientes para el diagnóstico ante mortem (Clarkson y Faull 1990, Komnenou 2000). El quiste normalmente presenta un crecimiento lento y a veces la sintomatología aparece sólo cuando el tamaño es ya grande. A la evaluación radiográfica simple no es posible encontrar signos radiológicos específicos indicativos de coenurosis cerebral ovina (Gonzalo-Orden y col 1999). Sin embargo, cuando las anormalidades radiográficas están presentes, estas son más evidentes en vistas posteroanteriores de cráneo (Tirgari 1987). Áreas radiolúcidas de forma creciente, triangular o rectangular a nivel del cráneo o desviación del septum nasal son signos radiográficos posibles de observar en casos de coenurosis cerebral ovina (Tirgari 1987). En la evaluación ultrasonográfica los quistes aparecen como estructuras anecoicas, redondas y/o como lesiones uniloculares (Doherty 1989a). Estudios de tomografía computarizada en seres humanos muestran los quistes como estructuras esféricas, hipodensas, que presentan un efecto de masa notorio (Tuzun y col 2002). En este estudio el quiste fue observado como una estructura hipodensa de aspecto circular incorporada en los ventrículos laterales. La radiopacidad del contenido quístico observada fue similar a la del líquido cefalorraquídeo, lo que concuerda con estudios previos hechos en seres humanos y ovinos (Pau y col 1987, Gonzalo-Orden y col 1999-2000). Autores indican que en las imágenes de TC craneales no contrastadas de humanos y ovinos, la pared del quiste puede ser

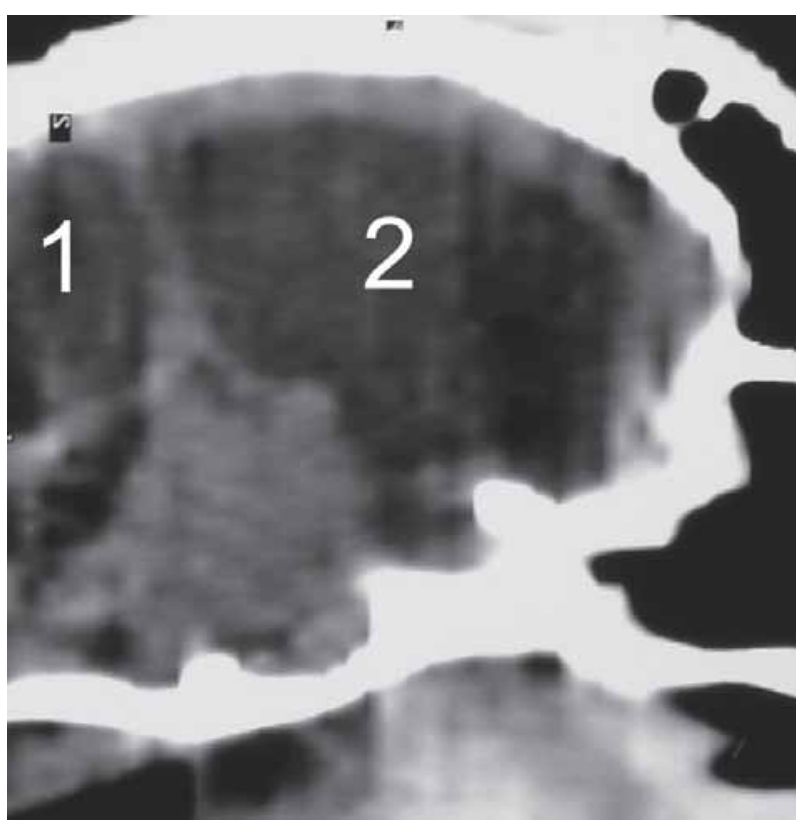

Figura 3. Reconstrucción multiplanar longitudinal de TC sin contraste de cráneo de oveja. A nivel de fosa caudal se observa el quiste parasitario (1). Rostralmente se observa ventriculomegalia a nivel de hemisferios cerebrales y adelgazamiento de corteza cerebral (2).

Non-contrast longitudinal multiplanar CT reconstruction of a sheep skull. A parasitic cyst is observed at the caudal fossa level (1). A ventriculomegaly is observed rostrally at the cerebral hemispheres and also narrowing of the cerebral cortex is observed (2). 
isodensa o hiperdensa al tejido cerebral (Gonzalo-Orden y col 1999, 2000). El anillo hiperdenso observado alrededor del quiste a nivel de fosa posterior en las imágenes de TC de este estudio se cree está asociado al engrosamiento de la cápsula fibrosa del quiste (Kramer y col 1989). En seres humanos con quistes parasíticos, edema periquístico usualmente no es observado en imágenes de TC aunque sí la presencia de efecto de masa (Tuzun y col 2002). La localización del quiste determinada por las imágenes de TC en este estudio permitió explicar parte de la signología neurológica, aunque algunos autores indican que la severidad de los signos clínicos puede no relacionarse con la cantidad y ubicación de los quistes (Skerrit y Stallbaumer 1984). En este reporte la ubicación del quiste fue a nivel de hemisferios cerebrales. Estudios previos indican que un $85 \%$ a un $96 \%$ de los quistes parasitarios poseen una ubicación prosencefálica y el resto una ubicación cerebelar, en tallo encefálico o médula espinal (Achenef 1999, Komnenou 2000). Aunque en este reporte las dimensiones del quiste fueron difíciles de delimitar, estudios previos indican que estos pueden medir alrededor de 0,5 a $10 \mathrm{~cm}$ de diámetro (Tirgari 1987, Biyikoglu 2003).

La presencia de reblandecimiento óseo a nivel del hueso frontal encontrado en este estudio ha sido informada previamente, autores indican que la presencia de dicho signo puede variar entre un 2 a un $42 \%$ de los casos de coenurosis cerebral ovina (Skerrit y Stallbaumer 1984, Komnenou y col 2000). Por tanto, este signo clínico no constituye un signo confiable en la localización del quiste, ya que el reblandecimiento es más bien producto del aumento generalizado de la presión intracraneana que debido al efecto compresivo local del quiste (Skerrit 1987, 1991).

Otras patologías nerviosas de carácter quístico que podrían presentar signos imagenológicos en la TC similares al de coenurosis cerebral en ovinos incluyen hidrocefalia congénita, hidrancefalia, holoprosencefalia, absceso piogénicos, ependimomas y síndrome de Dandy-Walker (Martin y Aitken 2000).

En conclusión la TC constituye una técnica no invasiva de gran utilidad en el diagnóstico de la coenurosis cerebral ovina no sólo en la entrega de información referente a la localización, tamaño y número de quistes parasitarios sino además permite estimar el grado de compromiso del encéfalo.

\section{RESUMEN}

El propósito de este estudio fue caracterizar, en una hembra ovina con signos clínicos de coenurosis cerebral, los hallazgos radiográficos observados mediante tomografía computarizada (TC). La TC de cráneo fue realizada sin contraste utilizando un escáner de cuarta generación. En las imágenes transversales de TC el quiste fue observado como una estructura hipodensa rodeada por un anillo hiperdenso y localizada en los hemisferios cerebrales a nivel del sistema ventricular. La presencia de ventriculomegalia y atrofia cortical indicaron la presencia de hidrocéfalo secundario. El Coenurus fue removido quirúrgicamente por trepanación a través del hueso frontal y luego de algunos días el animal recuperó su estado de salud. Los resultados observados indican que la TC constituye un método útil en demostrar la localización anatómica del quiste y en el planeamiento quirúrgico para la remoción de éste.

\section{REFERENCIAS}

Achenef M, T Markos, G Feseha, A Hibret, S Tembely. 1999. Coenurus cerebralis infection in Ethiopian highland sheep: incidence and observations on pathogenesis and clinical signs. Trop Anim Health Prod 31, 15-24.

Biyikoglu G, A Doganay. 1998. Effects praziquantel and albendazole on Coenurus cerebralis in experimental infected lambs. Turk $J$ Vet Anim Sci 22, 43-48.

Brewer B D. 1983. Neurologic disease of sheep and goats. Vet Clin North Am Large Anim Pract 5, 677-700.

Clarkson M, W Faull. 1990 Coenurosis. In: Handbook for the sheep clinician, Liverpool University Press, Liverpool, Pp 33-38.

Daly P J. 1985. Treatment of gid. Vet Rec 116, 59.

Doherty M L, H F Bassett, R Breathnach, M L Monaghan, B A McErlean. 1989'. Outbreak of acute coenuriasis in adult sheep in Ireland. Vet Rec 125, 185.

Doherty M L, H McAllister, A Healy. 1989 . Ultrasound as an aid to Coenurus cerebralis cyst localization in a lamb. Vet Rec 124, 591.

Dyson D A, K A Linklater.1979. Problems in the diagnosis of acute coenurosis in sheep. Vet Rec 104, 528-529.

Fraser G M. 1991. The Merck Veterinary Manual. Handbook of Diagnosis. Therapy and Disease Prevention and Control for Veterinarians. Merck and Co. Inc., Rahway, NJ, USA, Pp 604.

Gonzalo-Orden J M, A Diez, J R Altonaga, J M Gonzalo, M A Orden. 1999. Computed tomographic findings in ovine coenurosis. Vet Radiol \&Ultrasound 40, 441-444.

Gonzalo-Orden J M, J R Altonaga, A Diez, J M Gonzalo, M A Orden. 2000. Correlation between MRI, computed tomographic findings and clinical signs in a case of ovine coenurosis. Vet Rec 146, 352-353.

Gutiérrez R, J Inostroza, C Oberg, A Baquerizo, W Muñoz, W Eskuche, J Fierro, V Pineda, M Tager .1992. Hydatidosis in the IX $^{\text {th }}$ Region of Chile. A regional problem and challenge. Rev Med Chil 120, 311-316.

Ing M B, P M Schantz, J A Turner. 1998. Human coenurosis in North America: case reports and review. Clin Infect Dis 27, 519-523.

Komnenou A, S Argyroudis, N Giadinis, A Dessiris. 2000. Surgical treatment of coenurosis (gid) in sheep. Vet Rec 147, 242-244.

Lavin S, I Marco, J Pastor. 1995. Cerebral coeneurosis in chamois (Rupicapra pyrenaica). Zentralbl Veterinarmed B 42, 205-8.

Martin W B, I Aitken. 1997. Diseases of Sheep. Blackwell Science, Edinburgh, UK.

Mayhew I G. 1989. Large Animal Neurology: a Handbook for Veterinary Clinicians, Lea \& Febiger, Philadelphia, London, Pp 15-47.

Oryan A, N Moghaddar, S N Gaur. 1994. Metacestodes of sheep with special reference to their epidemiological status, pathogenesis and economic implications in Fars Province, Iran. Vet Parasitol 51, 231-40.

Pau A, S Turtas, M Brambilla, A Leoni, M Rosa, G L Viale. 1987. Computed tomography and magnetic resonance imaging of cerebral coenurosis. Surg Neurol 27, 548-552.

Skerritt G C, M F Stallbaumer. 1984. Diagnosis and treatment of coenuriasis (gid) in sheep. Vet Rec 115, 399-403.

Skerrit G C, N A Tadich. 1986. Tratamiento quirúrgico de coenurosis en ovinos. Arch Med Vet 18, 135-139.

Skerrit C G. 1987. New diagnostic and operative technique for gid. Proceedings of the Sheep Veterinary Society 12, 12-17. 
Skerrit C G. 1991. Coenuriasis. In: Diseases of sheep. $2^{\text {nd }}$ ed. Martin W B, Aitken I D (eds). Oxford Blackwell Scientific Publication, Pp 188-194.

Smith M C, C S Bailey, N Baker, N Kock. 1988. Cerebral coenurosis in a cat. J Am Vet Med Assoc 192, 82-84.

Soulsby E J L. 1982. Parasitología y enfermedades parasitarias en los animales domésticos. Nueva Editorial Interamericana, México DF, México.
Tirgari M, B R Howard, A Boargob. 1987. Clinical and radiographical diagnosis of coenurosis cerebralis in sheep and its surgical treatment. Vet Rec 120, 173-178.

Tuzun M, N Altinors, I S Arda, B Hekimoglu B. 2002. Cerebral hydatid disease CT and MR findings. Clin Imaging 26, 353-357.

Yoshiro T, E Momotani. 1988. A case of bovine coenurosis (Coenurus cerebralis) in Japan. Nippon Juigaku Zasshi 50, 433-438. 
